\title{
Truncated Cauchy Power Odd Fréchet-G Family of Distributions: Theory and Applications
}

\author{
M. Shrahili $\mathbb{I D}^{1}$ and I. Elbatal ${ }^{2}$ \\ ${ }^{1}$ Department of Statistics and Operations Research, College of Science, King Saud University, P. O. Box 2455, \\ Riyadh 11451, Saudi Arabia \\ ${ }^{2}$ Department of Mathematics and Statistics, College of Science, Imam Mohammad Ibn Saud Islamic University (IMSIU), \\ Riyadh, Saudi Arabia \\ Correspondence should be addressed to M. Shrahili; msharahili@ksu.edu.sa
}

Received 11 August 2021; Revised 3 October 2021; Accepted 5 October 2021; Published 27 October 2021

Academic Editor: Dan Selişteanu

Copyright $\odot 2021$ M. Shrahili and I. Elbatal. This is an open access article distributed under the Creative Commons Attribution License, which permits unrestricted use, distribution, and reproduction in any medium, provided the original work is properly cited.

\begin{abstract}
The truncated Cauchy power odd Fréchet-G family of distributions is presented in this article. This family's unique models are launched. Statistical properties of the new family are proposed, such as density function expansion, moments, incomplete moments, mean deviation, Bonferroni and Lorenz curves, and entropy. We investigate the maximum likelihood method for predicting model parameters of the new family. Two real-world datasets are used to show the importance and flexibility of the new family by using the truncated Cauchy power odd Fréchet exponential model as example of the family and compare it with some known models, and this model proves the importance and the flexibility for the new family.
\end{abstract}

\section{Introduction}

Many authors have lately made considerable attempts to create new families to expand well-known distributions and give flexible classes to represent data in a wide range of areas, including medical sciences, environmental sciences, engineering, demography, actuarial science, and economics. Many generic families have been developed and are used to explain a wide range of real-world events. Some examples of these families are beta-G [1], gamma-G [2], upper truncated Weibull distribution [3], and truncated Weibull-G more flexible and more reliable than beta-G distribution [4], truncated inverted Kumaraswamy-G by Bantan et al. [5], type II truncated FréchetG [6], type II power TL by Bantan et al. [7], odd generalized N-H by Ahmad et al. [8], Topp-Leone (TL) odd Fréchet-G by Al-Marzouki et al. [9], transmuted odd Fréchet-G by Badr et al. [10], and truncated Burr X-G by Bantan et al. [11], among others.
Haq and Elgarhy [12] proposed the odd Fréchet-G (OF, $-G$ ) with cumulative function (cdf) as follows, for $x>0$,

$$
H(x ; \alpha, \xi)=e^{-(\bar{G}(x, \xi) / G(x, \xi))^{\alpha}}, \quad \alpha>0,
$$

and probability density function (pdf) is

$$
h(x ; \alpha, \xi)=\frac{\alpha g(x, \xi) \bar{G}(x, \xi)^{\alpha-1}}{G(x, \xi)^{\alpha+1}} e^{-(\bar{G}(x, \xi) / G(x, \xi))^{\alpha}} .
$$

The Cauchy (C) distribution plays an important role and has applications in different fields such as econometrics, engineering, spectroscopy, biological analysis, reliability, queueing theory, and stochastic modeling of decreasing hazard rate life devices. There are many authors who have been displayed various generalization and extension forms of Cauchy distribution in the statistical literature, for examples, Rider [13] presented generalized C distribution, A truncated $\mathrm{C}$ distribution by Nadarajah et al. [14], the existence of the moments of the $\mathrm{C}$ distribution by Ohakwe and 
Osu [15], Jacob and Jayakumar[16] studied half-C distribution, Kumaraswamy-half-C distribution by Hamedani and Ghosh [17], Alshawarbeh and Famoye [18] presented properties of beta- $\mathrm{C}$ distribution, and the power- $\mathrm{C}$ negativebinomial distribution by Zubair et al. [19], among others.

Recently, Aldahlan et al. [20] proposed the truncated C power-G (TCP-G) family. The cdf of the TCP-G family is given by

$$
F(x ; \lambda)=\frac{4}{\pi} \arctan (H(x))^{\lambda}, \quad x \in R,
$$

where $\lambda>0$. The corresponding pdf is

$$
f(x ; \lambda)=\frac{4 \lambda h(x)(H(x))^{\lambda-1}}{\pi\left[1+(H(x))^{2 \lambda}\right]} .
$$

The aim of this article is to provide a new, broader, and more flexible family of distributions based on the odd Fréchet-G and truncated $\mathrm{C}$ power-G (TCP-G) families. The new proposed family has many wide applications in physics, medicine, engineering, and finance. We construct a new family called TCP odd Fréchet-G (TCPOF $-G$ ) family of distributions by inserting equation (1) into equation (3); the cdf and pdf of the TCPOF $-G$ are

$$
F(x ; \lambda, \alpha, \xi)=\frac{4}{\pi} \arctan e^{-\lambda(\bar{G}(x, \xi) / G(x, \xi))^{\alpha}}, \quad x>0,
$$

and

$$
\begin{aligned}
f(x ; \lambda, \alpha, \xi)= & \frac{4 \lambda \alpha g(x, \xi) \bar{G}(x, \xi)^{\alpha-1}}{\pi G(x, \xi)^{\alpha+1}} e^{-\lambda(\bar{G}(x, \xi) / G(x, \xi))^{\alpha}}, \\
& \times\left[1+e^{-2 \lambda\left((\bar{G}(x, \xi) / G(x, \xi))^{\alpha}\right)^{\alpha}}\right]^{-1} .
\end{aligned}
$$

Henceforward, a random variable $X$ having pdf equation (6) will be defined as $X \sim \operatorname{TCPOF}(\lambda, \alpha, \xi)$. The survival and hazard rate functions for the TCPOF $-G$ family are by

The quantile function (qf) of TCPOF-G family is given

$$
F^{-1}(u)=Q_{G}(u)=G^{-1}\left[\frac{1}{1+\{-(1 / \lambda) \log [\tan (u \pi / 4)]\}^{1 / \alpha}}\right] .
$$

The median is given by

$$
M=Q_{2}=Q(0.5)=G^{-1}\left[\frac{1}{1+\{-(1 / \lambda) \log [\tan (0.5 \pi / 4)]\}^{1 / \alpha}}\right] \text {. }
$$

The structure of this paper is as follows. Section 2 has a useful linear explanation of the TCPOF density as well as several specific models. Section 3 looks at structural properties of the TCPOF-G. In Section 4, we discuss the suggested family's entropy. The maximum likelihood method is used to estimate the model parameters in Section 5. Section 6 provides applications to real-world datasets to illustrate the proposed family's flexibility.

\section{Linear Representation of TCPOF}

If $|z|<1$ and $b>0$ is a real noninteger, then the following power series hold:

$$
(1+z)^{-b}=\sum_{k=0}^{\infty}\left(\begin{array}{c}
-b \\
k
\end{array}\right) z^{k}
$$

Applying equation (10) in equation (6), we get

$$
f_{\mathrm{TCPOF}_{r}}(x ; \lambda, \alpha, \xi)=\frac{4 \lambda \alpha g(x, \xi) \bar{G}(x, \xi)^{\alpha-1}}{\pi G(x, \xi)^{\alpha+1}} \sum_{i=0}^{\infty}(-1)^{i} e^{-\lambda(2 i+1)(\bar{G}(x, \xi) / G(x, \xi))^{\alpha}} .
$$

By using the power series for the exponential function, the last term in equation (11) gives

$$
e^{-\lambda(2 i+1)(\bar{G}(x, \xi) / G(x, \xi))^{\alpha}}=\sum_{j=0}^{\infty} \frac{(-1)^{j} \lambda^{j}(2 i+1)^{j}}{j !} \frac{\bar{G}(x, \xi)^{\alpha j}}{G(x, \xi)^{\alpha j}}
$$


The TCPOF $-G$ density reduces to

$$
f_{\mathrm{TCPOF}_{r}}(x ; \lambda, \alpha, \xi)=\frac{4 \alpha g(x, \xi)}{\pi} \sum_{i, j=0}^{\infty} \frac{(-1)^{i+j} \lambda^{j+1}(2 i+1)^{j}}{j !} \frac{\bar{G}(x, \xi)^{\alpha(j+1)-1}}{G(x, \xi)^{\alpha(j+1)+1}} .
$$

Using the general binomial expansion, we can write

$$
G(x, \xi)^{-(\alpha(j+1)+1)}=\sum_{k=0}^{\infty} \frac{\Gamma(\alpha(j+1)+k+1)}{k ! \Gamma(\alpha(j+1)+1)}(1-G(x, \xi))^{k},
$$

$$
(1-G(x, \xi))^{\alpha(j+1)+k-1}=\sum_{m=0}^{\infty}(-1)^{m} \frac{\Gamma(\alpha(j+1)+k)}{m ! \Gamma(\alpha(j+1)+k-m)} G(x, \xi)^{m} .
$$

Inserting equations (14) and (15) in equation (13), the TCPOF $-G$ density becomes

$$
f_{\mathrm{TCPOF}_{r}}(x ; \lambda, \alpha, \xi)=\sum_{m=0}^{\infty} \psi_{m} \pi_{(m+1)}(x),
$$

where $h_{v}(x)=v g(x ; \xi) G(x ; \xi)^{v-1}$ denotes the pdf of the exponentiated generalized (Exp-G) distribution with power parameter $\nu$, and

$$
\begin{aligned}
\psi_{m}= & \frac{4 \alpha}{\pi} \sum_{i, j, k=0}^{\infty} \frac{(-1)^{i+j+m} \lambda^{j+1}(2 i+1)^{j}}{j ! k ! m !(m+1)} \frac{\Gamma(\alpha(j+1)+k+1)}{\Gamma(\alpha(j+1)+1)} \\
& \times \frac{\Gamma(\alpha(j+1)+k)}{m ! \Gamma(\alpha(j+1)+k-m)} .
\end{aligned}
$$

2.1. Four Special Models of the TCPOF Family. In this part, we presented three distinct models of the TCPOF family of distributions. When the cdf $G(x)$ and pdf $g(x)$ have simple analytic expressions, the pdf equation (8) will be most tractable. Based on the baseline distributions, we propose four submodels of this family: Weibull, exponential, Rayleigh, and Lomax. The cdf and pdf files for these baseline models are provided in Table 1.

2.1.1. TCPOF Weibull (TCPOFW) Distribution. The cdf and pdf of TCPOFW distribution are

$$
\begin{aligned}
& F(x ; \lambda, \alpha, \mu, \beta)=\frac{4}{\pi} \arctan e^{\left.\left.-\lambda\left(e^{-\beta \beta x}\right)^{\mu} / 1-e^{-\beta \beta x}\right)^{\mu}\right)^{\alpha}}, \quad x>0,
\end{aligned}
$$

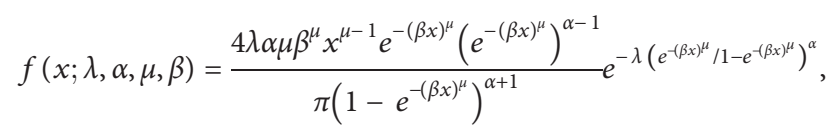

$$
\begin{aligned}
& {\left[1+e^{-2 \lambda\left(e^{-(\beta x)^{\mu}} / 1-e^{-(\beta x)^{\mu}}\right)^{\alpha}}\right]^{-1} .}
\end{aligned}
$$

2.1.2. TCPOF Exponential (TCPOFE) Distribution. The cdf and pdf of the TCPOFE model (for $x>0$ ) are

$$
\begin{aligned}
F(x ; \lambda, \alpha, \beta)= & \frac{4}{\pi} \arctan e^{-\lambda\left(e^{-\beta x} / 1-e^{-\beta x}\right)^{\alpha}}, \quad x>0, \\
f(x ; \lambda, \alpha, \beta)= & \frac{4 \lambda \alpha \beta e^{-\beta x}\left(e^{-\beta x}\right)^{\alpha-1}}{\pi\left(1-e^{-\beta x}\right)^{\alpha+1}} \\
& e^{-\lambda\left(e^{-\beta x} / 1-e^{-\beta x}\right)^{\alpha}}, \\
& {\left[1+e^{-2 \lambda\left(e^{-\beta x} / 1-e^{-\beta x}\right)^{\alpha}}\right]^{-1} . }
\end{aligned}
$$

2.1.3. TCPOF Rayleigh (TCPOFR) Distribution. The cdf and pdf of the TCPOFR model are

$$
\begin{aligned}
F(x ; \lambda, \alpha, \beta)= & \frac{4}{\pi} \arctan e^{-\lambda\left(e^{-(\beta x)^{2}} / 1-e^{-(\beta x)^{2}}\right)^{\alpha}}, \quad x>0, \\
f(x ; \lambda, \alpha, \beta)= & \frac{4 \lambda \alpha \mu \beta^{2} x e^{-(\beta x)^{2}}\left(e^{-(\beta x)^{2}}\right)^{\alpha-1}}{\pi\left(1-e^{-(\beta x)^{2}}\right)^{\alpha+1}} \\
& e^{-\lambda\left(e^{-(\beta x)^{2}} / 1-e^{-(\beta x)^{2}}\right)^{\alpha}}, \\
& {\left[1+e^{2-\lambda\left(e^{-(\beta x)^{2}} / 1-e^{-(\beta x)^{2}}\right)^{\alpha}}\right]^{-1} . }
\end{aligned}
$$

2.1.4. TCPOF Lomax (TCPOFL) Distribution. The cdf and pdf of the TCPOFL model are 
TABLE 1: Some new models of the new family.

\begin{tabular}{lccc}
\hline Model & $G(x ; \xi)$ & $g(x ; \xi)$ & $\bar{G}(x ; \xi) / G(x ; \xi)$ \\
\hline Weibull & $1-e^{-(\beta x)^{\mu}}$ & $\mu \beta^{\mu} x^{\mu-1} e^{-(\beta x)^{\mu}}$ & $e^{-(\beta x)^{\mu}} / 1-e^{-(\beta x)^{\mu}}$ \\
$|z|<1$ & $1-e^{-\beta x}$ & $\beta e^{-\beta x}$ & $e^{-\beta x} / 1-e^{-\beta x}$ \\
Rayleigh & $1-e^{-(\beta x)^{2}}$ & $\beta^{2} x e^{-(\beta x)^{2}}$ & $e^{-(\beta x)^{2} / 1-e^{-(\beta x)^{2}}}$ \\
Lomax & $1-(1+(x / b))^{-a}$ & $a / b(1+(x / b))^{-a-1}$ & $(1+(x / b))^{-a} / 1-(1+(x / b))^{-a}$ \\
\hline
\end{tabular}

$$
\begin{aligned}
F(x ; \lambda, \alpha, a, b)= & \frac{4}{\pi} \arctan e^{-\lambda\left((1+(x / b))^{-a} / 1-(1+(x / b))^{-a}\right)^{\alpha}}, \quad x>0 \\
f(x ; \lambda, \alpha, a, b)= & \frac{4 \lambda \alpha(a / b)(1+(x / b))^{-a-1}\left((1+(x / b))^{-a}\right)^{\alpha-1}}{\pi\left(1-(1+(x / b))^{-a}\right)^{\alpha+1}} \\
& e^{-\lambda\left((1+(x / b))^{-a} / 1-(1+(x / b))^{-a}\right)^{\alpha}} \\
& \times\left[1+e^{-2 \lambda\left((1+(x / b))^{-a} / 1-(1+(x / b))^{-a}\right)^{\alpha}}\right]^{-1}
\end{aligned}
$$

Plots of the TCPOFW, TCPOFE, TCPOFR, and TCPOFL densities are represented in Figure 1.

\section{Structural Properties}

Our study focused on ordinary moments, incomplete moments (IMs), moment generating functions (MGFs), mean deviations (MD), Lorenz and Bonferroni curves (LBCs), and residual life (RL) functions.

3.1. Ordinary Moments and Incomplete Moments Functions. A first formula for the $r_{\text {th }}$ ordinary moment of $X$, say $\mu_{r}^{\prime}$, is

$$
\mu_{r}^{\prime}=E\left(X^{r}\right)=\sum_{m=0}^{\infty} \psi_{m} E\left(Y_{(m+1)}^{r}\right) \text {. }
$$

A second formula depending on the quantile function can be written as $E\left(X^{r}\right)=(m+1) \int_{-\infty}^{\infty} g(x) G(x)^{m} \mathrm{~d} x=$ $(m+1) \int_{0}^{1} Q_{G}(u ; \xi)^{r} u^{d} \mathrm{~d} u$. The $s_{t h}$ IMs of $X$ defined by $v_{s}(t)$ for any real $s>0$ can be expressed from equation (16) as

$$
\nu_{s}(t)=\int_{-\infty}^{t} x^{s} f(x) \mathrm{d} x=\sum_{m=0}^{\infty} \psi_{m} \int_{-\infty}^{t} x^{s} \pi_{(m+1)}(x) \mathrm{d} x .
$$

Equation (23) denotes the $s_{\text {th }}$ IMs of $\pi_{(m+1)}$. The MDs about the mean $\mu=E(X)$ and the MDs about the median $M$ are defined by

$$
\begin{aligned}
& \delta_{1}(x)=E\left|X-\mu_{1}^{\prime}\right|=2 \mu_{1}^{\prime} F\left(\mu_{1}^{\prime}\right)-2 \nu_{1}\left(\mu_{1}^{\prime}\right), \\
& \delta_{2}(x)=E|X-M|=\mu_{1}^{\prime}-2 \nu_{1}(M),
\end{aligned}
$$

respectively, where $\mu_{1}^{\prime}=E(X), M=\operatorname{median}(X)=Q(1 / 2)$, $F\left(\mu_{1}^{\prime}\right)$ is evaluated from equation (5), and $\nu_{1}(t)$ is the first IM given by equation (23) with $s=1$, where

$$
\begin{aligned}
v_{1}(t) & =\int_{-\infty}^{t} x f(x) \mathrm{d} x \\
& =\sum_{m=0}^{\infty} \psi_{m} \int_{-\infty}^{t} x \pi_{(m+1)}(x) \mathrm{d} x .
\end{aligned}
$$

We can determine $\delta_{1}(x)$ and $\delta_{2}(x)$ by two techniques, the first can be obtained from equation (16) as $v_{1}(t)=\sum_{m=0}^{\infty} \psi_{m} Y_{(m+1)}(t)$ where $Y_{(m+1)}(t)=\int_{-\infty}^{t} x \pi_{(m+1)}$ $(x) \mathrm{d} x$ is the first IM of the Exp-G distribution. The second technique is given by $\nu_{1}(t)=\sum_{m=0}^{\infty} \psi_{m} \delta_{(m+1)}(t)$ where

$$
\delta_{(m+1)}(t)=(m+1) \int_{0}^{G(t)} u^{(m+1)} Q_{G}(u) \mathrm{a} u
$$

For a positive random variable $X$, the LBCs, for a given probability $p$, are given by $L(p)=\left(1 / \mu_{1}^{\prime}\right) \nu_{1}(q)$ and $B(p)=\left(1 / p \mu_{1}^{\prime}\right) \nu_{1}(q)$, respectively, where $\mu_{1}^{\prime}=E(X)$, and $q=Q(p)$ is the quantile function of $X$ at $p$.

\subsection{Moment Generating Function. The MGF of $X$ is}

$$
M_{X}(t)=E\left(e^{t X}\right)=\sum_{m=0}^{\infty} \psi_{m} M_{(m+1)}(t)
$$

where $M_{(m+1)}(t)$ denotes the MGF of $\pi_{(m+1)}$.

A second alternative formula can be derived from equation (16) as follows: $M_{X}(t)=\sum_{m=0}^{\infty} \psi_{m} \gamma(t, m+1)$, where $\gamma(t, m+1)=(m+1) \int_{0}^{1} u^{m} e^{t Q_{G}(u)} \mathrm{d} u$.

3.3. Moments of RL and Reversed $R L$. The $r^{\text {th }}$ order moment of the RL is given by 

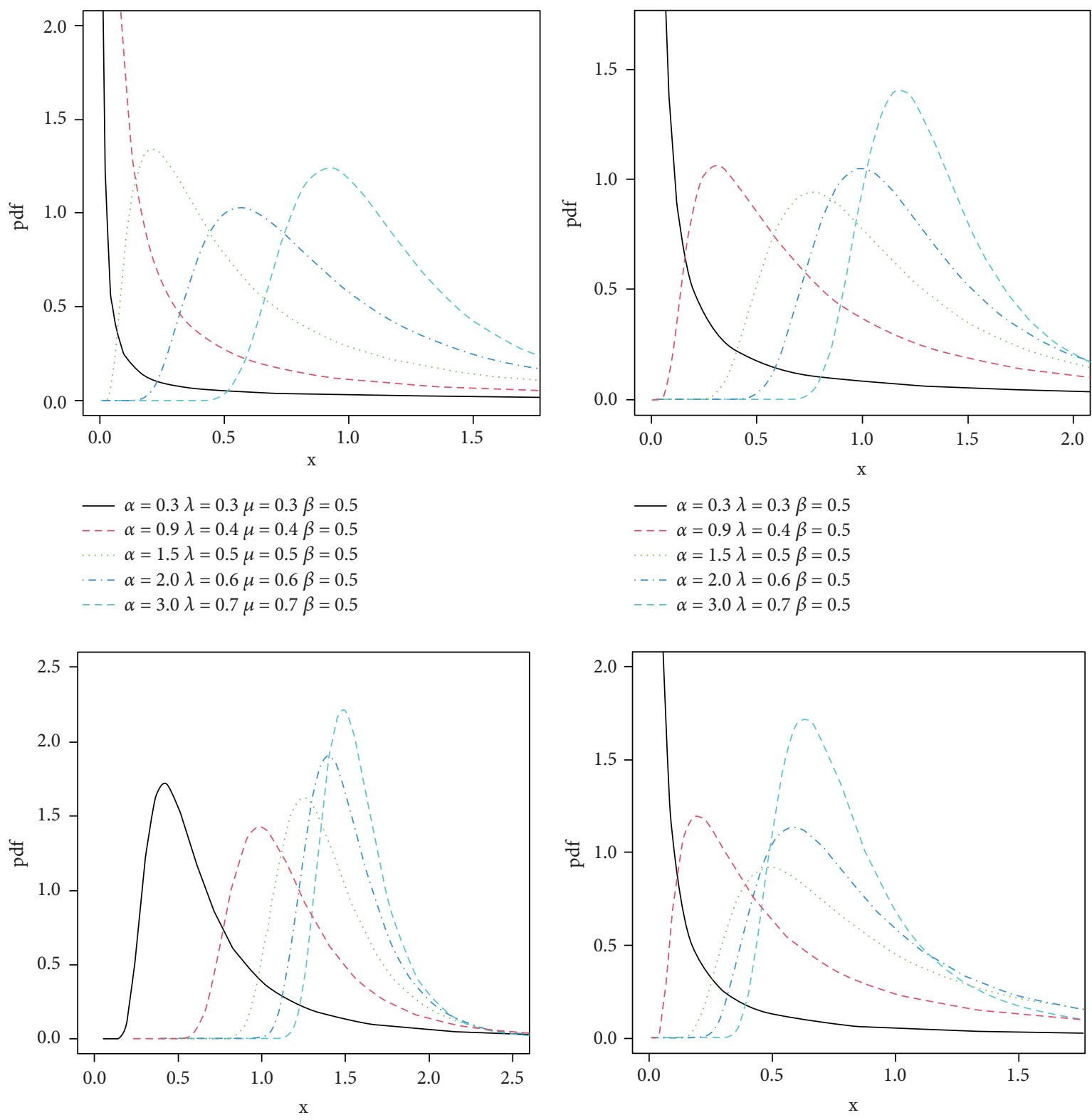

$\begin{aligned} &-\alpha=0.9 \lambda=0.1 \beta=0.5 \\ &--\alpha=1.5 \lambda=0.2 \beta=0.5 \\ & \cdots \alpha=2.0 \lambda=0.3 \beta=0.5 \\ &-\alpha \alpha=2.5 \lambda=0.4 \beta=0.5 \\ &--\alpha=3.0 \lambda=0.5 \beta=0.5\end{aligned}$

$$
\begin{aligned}
&-\alpha=0.3 \lambda=0.3 \mathrm{a}=0.3 \mathrm{~b}=0.5 \\
&--\alpha=0.9 \lambda=0.4 \mathrm{a}=0.4 \mathrm{~b}=0.5 \\
& \cdots-\alpha=1.5 \lambda=0.5 \mathrm{a}=0.5 \mathrm{~b}=0.5 \\
&--\alpha=2.0 \lambda=0.6 \mathrm{a}=0.6 \mathrm{~b}=0.5 \\
&--\alpha=3.0 \lambda=0.7 \mathrm{a}=0.7 \mathrm{~b}=0.5
\end{aligned}
$$

FIgUre 1: TCPOFW, TCPOFE, TCPOFR, and TCPOFL densities for different values of parameters.

$$
\begin{aligned}
\psi_{r}(t) & =E\left((X-t)^{r} \mid X>t\right)=\frac{1}{\bar{F}(t)} \int_{t}^{\infty}(x-t)^{r} f(x) \mathrm{d} x \quad, r \geq 1 \\
& =\frac{1}{\bar{F}(t)} \sum_{m=0}^{\infty} \psi_{m}^{*} \int_{t}^{\infty} x^{r} \pi_{(m+1)}(x) \mathrm{d} x,
\end{aligned}
$$

where $\psi_{m}^{*}=\sum_{m=0}^{\infty} \sum_{m=0}^{r} \psi_{m}\left(\begin{array}{c}r \\ m\end{array}\right)(-t)^{r-m}$. The mean RL (MRL) of TCPOF, $-G$ family can be obtained by setting $r=$ 1 in equation (28), defined as

$$
\psi_{1}(t)=E\left(X_{t}\right)=E(X \mid X>t)
$$

The $r^{\text {th }}$ order moment of the reversed RL (or inactivity time) can be obtained by the next equation 


$$
\begin{aligned}
m_{r}(t) & =E\left((t-X)^{r} \mid X \leq t\right)=\frac{1}{F(t)} \int_{0}^{t}(t-x)^{r} f(x) \mathrm{d} x, \quad r \geq 1 \\
& =\frac{1}{F(t)} \sum_{m=0}^{\infty} \psi_{m}^{*} \int_{0}^{t} x^{r} \pi_{(m+1)}(x) \mathrm{d} x .
\end{aligned}
$$

\section{Entropy}

The Rényi entropy is defined by $(\theta>0, \theta \neq 1$

$$
I_{R}(\theta)=\frac{1}{1-\theta} \log \left[\int_{-\infty}^{\infty} f^{\theta}(x) \mathrm{d} x\right] .
$$

Using the general binomial expansion, applying the same method of the linear representation equation (16) and after some simplifications, we get

$$
I_{R}(\theta)=\frac{1}{1-\theta} \log \left[\int_{-\infty}^{\infty} f^{\theta}(x) \mathrm{d} x\right],
$$

where

$$
\begin{aligned}
\Delta_{m}= & \left(\frac{4 \lambda \alpha}{\pi}\right)^{\theta} \sum_{i, j, k=0}^{\infty} \frac{(-1)^{i+j+m} \lambda^{j}(2 i+\theta)^{j}}{i ! j ! k ! m !} \\
& \times \frac{\Gamma(\theta+i) \Gamma(\alpha(\theta+j)+k+\theta) \Gamma(\alpha(\theta+j)+k-\theta+1)}{\Gamma(\alpha(\theta+j)+\theta) \Gamma(\alpha(\theta+j)+k-\theta-m+1)} .
\end{aligned}
$$

\section{Maximum Likelihood Estimation}

Let $x_{1}, \ldots, x_{n}$ be a random sample of size $n$ from the TCPOF $-G$ given by equation (11). Let $\Omega=(\lambda, \alpha, \xi)^{T}$ be $q \times$ 1 vector of parameters. The log-likelihood function is

$$
\begin{aligned}
L_{n}= & n \log \left(\frac{4 \lambda}{\pi}\right)+n \log (\alpha)+\sum_{i=1}^{n} \log g\left(x_{i} ; \xi\right) \\
& +(\alpha-1) \sum_{i=1}^{n} \log \bar{G}\left(x_{i} ; \xi\right) \\
& -(\alpha+1) \sum_{i=1}^{n} \log \left(G\left(x_{i} ; \xi\right)\right)-\lambda \sum_{i=1}^{n} d_{i}^{\alpha} \\
& -\sum_{i=1}^{n} \log \left\{1+e^{-2 \lambda d_{i}^{\alpha}}\right\} .
\end{aligned}
$$

$d_{i}=\bar{G}\left(x_{i} ; \xi\right) / G\left(x_{i} ; \xi\right)$. The score vector components, say, $U(\Omega)=\partial L_{n} / \partial \Omega=\left(\partial L_{n} / \partial \lambda, \partial L_{n} / \partial \alpha, \partial L_{n} / \partial \xi\right)$ are given by

Thus, Rényi entropy of TCPOF, $-G$ family is given by

$$
I_{R}(\theta)=\frac{1}{1-\theta} \log \left\{\sum_{m=0}^{\infty} \Delta_{m} \int_{-\infty}^{\infty} g(x)^{\theta} G(x)^{m} \mathrm{~d} x\right\} \text {. }
$$

$$
\begin{aligned}
U_{\lambda}= & \frac{\partial L_{n}}{\partial \lambda}=\frac{n}{\lambda}-\sum_{i=1}^{n} d_{i}^{\alpha}+\sum_{i=1}^{n} \frac{2 d_{i}^{\alpha} e^{-2 \lambda d_{i}^{\alpha}}}{1+e^{-2 \lambda d_{i}^{\alpha}}}, \\
U_{\alpha}= & \frac{\partial L_{n}}{\partial \alpha}=\frac{n}{\alpha}+\sum_{i=1}^{n} \log \bar{G}\left(x_{i} ; \xi\right)-\sum_{i=1}^{n} \log \left(G\left(x_{i} ; \xi\right)\right) \\
& -\lambda \sum_{i=1}^{n} d_{i}^{\alpha} \log d_{i}+\sum_{i=1}^{n} \frac{2 \lambda d_{i}^{\alpha} e^{-2 \lambda d_{i}^{\alpha}} \log d_{i}}{1+e^{-2 \lambda d_{i}^{\alpha}}}, \\
U_{\xi}= & \frac{\partial L_{n}}{\partial \xi_{k}}=\sum_{i=1}^{n} \frac{g^{\prime}\left(x_{i} ; \xi\right)}{g\left(x_{i} ; \xi\right)}+(\alpha-1) \sum_{i=1}^{n} \frac{\bar{G} \prime\left(x_{i} ; \xi\right)}{\bar{G}\left(x_{i} ; \xi\right)}-(\alpha+1) \sum_{i=1}^{n} \frac{G^{\prime}\left(x_{i} ; \xi\right)}{G\left(x_{i} ; \xi\right)} \\
& -\lambda \alpha \sum_{i=1}^{n} d_{i}^{\alpha-1}\left(\frac{\partial d_{i}}{\partial \xi_{k}}\right)+\sum_{i=1}^{n} \frac{2 \lambda \alpha d_{i}^{\alpha-1} e^{-2 \lambda d_{i}^{\alpha}}}{1+e^{-2 \lambda d_{i}^{\alpha}}}\left(\frac{\partial d_{i}}{\partial \xi_{k}}\right),
\end{aligned}
$$


TABle 2: Estimates for dataset I.

\begin{tabular}{|c|c|}
\hline Distributions & MLEs and SEs \\
\hline $\operatorname{TCPOFE}(\lambda, \alpha, \beta)$ & $\begin{array}{c}0.151,6.011,8.084 \\
(0.168),(1.024),(0.6141) \\
\end{array}$ \\
\hline $\operatorname{MOE}(a, b)$ & $\begin{array}{c}8.780,1.380 \\
(3.560),(0.194) \\
\end{array}$ \\
\hline $\operatorname{BrXE}(a, \beta)$ & $\begin{array}{c}0.475,0.2055 \\
(0.060),(0.012) \\
\end{array}$ \\
\hline $\operatorname{KE}(a, b, \beta)$ & $\begin{array}{c}3.3041,1.1002,1.0371 \\
(1.1061),(0.7642),(0.6141)\end{array}$ \\
\hline $\operatorname{GMOE}(a, b, \beta)$ & $\begin{array}{c}0.1789,47.6350,4.4652 \\
(0.0702),(44.9011),(1.3270) \\
\end{array}$ \\
\hline $\mathrm{BE}(a, b, \beta)$ & $\begin{array}{c}0.8073,3.4612,1.3311 \\
(0.6961),(1.0032),(0.8551)\end{array}$ \\
\hline $\operatorname{KMOE}(\alpha, a, b, \beta)$ & $\begin{array}{c}0.3731,3.4782,3.3063,0.2990 \\
(0.1358),(0.862),(0.781),(1.113)\end{array}$ \\
\hline $\operatorname{MOKE}(\alpha, a, b, \beta)$ & $\begin{array}{c}0.0081,2.7162,1.9861,0.0992 \\
(0.0021),(1.3158),(0.7839),(0.0481)\end{array}$ \\
\hline $\operatorname{ME}(\beta)$ & $\begin{array}{c}0.9252 \\
(0.0768) \\
\end{array}$ \\
\hline $\mathrm{E}(\beta)$ & $\begin{array}{c}0.540 \\
(0.063) \\
\end{array}$ \\
\hline
\end{tabular}

TABLE 3: Estimates for dataset II.

\begin{tabular}{|c|c|}
\hline Distributions & MLEs and SEs \\
\hline $\operatorname{TCPOFE}(\lambda, \alpha, \beta)$ & $\begin{array}{c}1.475,21.125,1.295 \\
(5.068),(0.990),(3.816)\end{array}$ \\
\hline $\operatorname{MOE}(a, b)$ & $\begin{array}{c}54.470,2.320 \\
(35.580),(0.370)\end{array}$ \\
\hline $\operatorname{BrXE}(a, \beta)$ & $\begin{array}{c}1.1635,0.3207 \\
(0.330),(0.030)\end{array}$ \\
\hline $\mathrm{KE}(a, b, \beta)$ & $\begin{array}{c}83.7558,0.5679,3.3329 \\
(42.3612),(0.3261),(1.1880)\end{array}$ \\
\hline $\operatorname{GMOE}(a, b, \beta)$ & $\begin{array}{c}0.5192,89.4623,3.1691 \\
(0.2561),(66.2782),(0.7721)\end{array}$ \\
\hline $\mathrm{BE}(a, b, \beta)$ & $\begin{array}{c}81.6333,0.5421,3.5142 \\
(120.4104),(0.3272),(1.4101)\end{array}$ \\
\hline $\operatorname{KMOE}(\alpha, a, b, \beta)$ & $\begin{array}{c}8.8679,34.8258,0.2989,4.8988 \\
(9.1459),(22.3119),(0.2387),(3.1757)\end{array}$ \\
\hline $\operatorname{MOKE}(\alpha, a, b, \beta)$ & $\begin{array}{c}0.1333,33.2322,0.5711,1.6691 \\
(0.3320),(57.8371),(0.7211),(1.8141)\end{array}$ \\
\hline $\operatorname{ME}(\beta)$ & $\begin{array}{c}0.9502 \\
(0.1501)\end{array}$ \\
\hline $\mathrm{E}(\beta)$ & $\begin{array}{c}0.526 \\
(0.117)\end{array}$ \\
\hline
\end{tabular}

Table 4: Some IC for dataset I.

\begin{tabular}{lcccccc}
\hline Distributions & $Z 1$ & $Z 2$ & $Z 3$ & $Z 4$ & $Z 7$ & $Z 8$ \\
\hline TCPOFE & 191.815 & 191.387 & 192.168 & 194.534 & 0.085 & $(0.674)$ \\
MOE & 210.36 & 214.92 & 210.53 & 212.16 & 0.10 & $(0.430)$ \\
BrXE & 235.30 & 239.90 & 235.50 & 237.10 & 0.22 & $(0.002)$ \\
KE & 209.42 & 216.24 & 209.77 & 212.12 & 0.09 & $(0.500)$ \\
GMOE & 210.54 & 217.38 & 210.89 & 213.24 & 0.09 & $(0.510)$ \\
BE & 207.38 & 214.22 & 207.73 & 210.08 & 0.11 & $(0.340)$ \\
KMOE & 207.82 & 216.94 & 208.42 & 211.42 & 0.09 & $(0.530)$ \\
MOKE & 209.44 & 218.56 & 210.04 & 213.04 & 0.10 & $(0.440)$ \\
ME & 210.40 & 212.68 & 210.45 & 211.30 & 0.14 & $(0.130)$ \\
E & 234.63 & 236.91 & 234.68 & 235.54 & 0.27 & $(0.060)$ \\
\hline
\end{tabular}

TABle 5: Some IC for dataset II.

\begin{tabular}{lcccccc}
\hline Distributions & $Z 1$ & $Z 2$ & $Z 3$ & $Z 4$ & $Z 7$ & $Z 8$ \\
\hline TCPOFE & 37.485 & 35.388 & 38.985 & 38.068 & 0.108 & $(0.975)$ \\
MOE & 43.51 & 45.51 & 44.22 & 43.90 & 0.18 & $(0.55)$ \\
BrXE & 48.10 & 50.10 & 48.80 & 48.50 & 0.25 & $(0.17)$ \\
KE & 41.78 & 44.75 & 43.28, & 42.32 & 0.14 & $(0.86)$ \\
GMOE & 42.75 & 45.74 & 44.25 & 43.34 & 0.15 & $(0.78)$ \\
BE & 43.48 & 46.45 & 44.98 & 44.02 & 0.16 & $(0.80)$ \\
KMOE & 42.80 & 46.84 & 45.55 & 43.60 & 0.15 & $(0.86)$ \\
MOKE & 41.58 & 45.54 & 44.25 & 42.30 & 0.14 & $(0.87)$ \\
ME & 54.32 & 55.31 & 54.54 & 54.50 & 0.32 & $(0.07)$ \\
E & 67.67 & 68.67 & 67.89 & 67.87 & 0.44 & $(0.004)$ \\
\hline
\end{tabular}

where $g^{\prime}\left(x_{i} ; \xi\right)=\partial g\left(x_{i} ; \delta\right) / \partial \xi_{k}, G^{\prime}\left(x_{i} ; \xi\right)=\partial G\left(x_{i} ; \xi\right) / \partial \xi_{k}$, and $\bar{G} \prime\left(x_{i} ; \xi\right)=\partial \bar{G}\left(x_{i} ; \xi\right) / \partial \xi_{k}$. The maximum likelihood estimation (MLE) of parameters is obtained by setting $\partial L_{n} / \partial \lambda=\partial L_{n} / \partial \alpha=\partial L_{n} / \partial \xi_{k}=0$ and solving these equations simultaneously to get theMLE $(\widehat{\Omega})$.

\section{Applications}

In this section, we used two datasets taken from Bjerkedal [21] and Gross and Clark [22]. The TCPOFE model as example of the new family is compared with some other known competitive models to demonstrate its importance in data modeling. The MLE method is used to estimate the parameters of the competitive models. Some information criterions (IC) are as follows: the Akaike IC (Z1), Bayesian IC (Z2), consistent Akaike IC (Z3), Hannan-Quinn IC (Z4), Cramér-Von Mises (Z5), Anderson-Darling (Z6), Kolmogorov-Smirnov (Z7) statistics with its $p$ value $(Z 8)$ model selection criteria, and goodness of fit tests are used to identify the best model. 

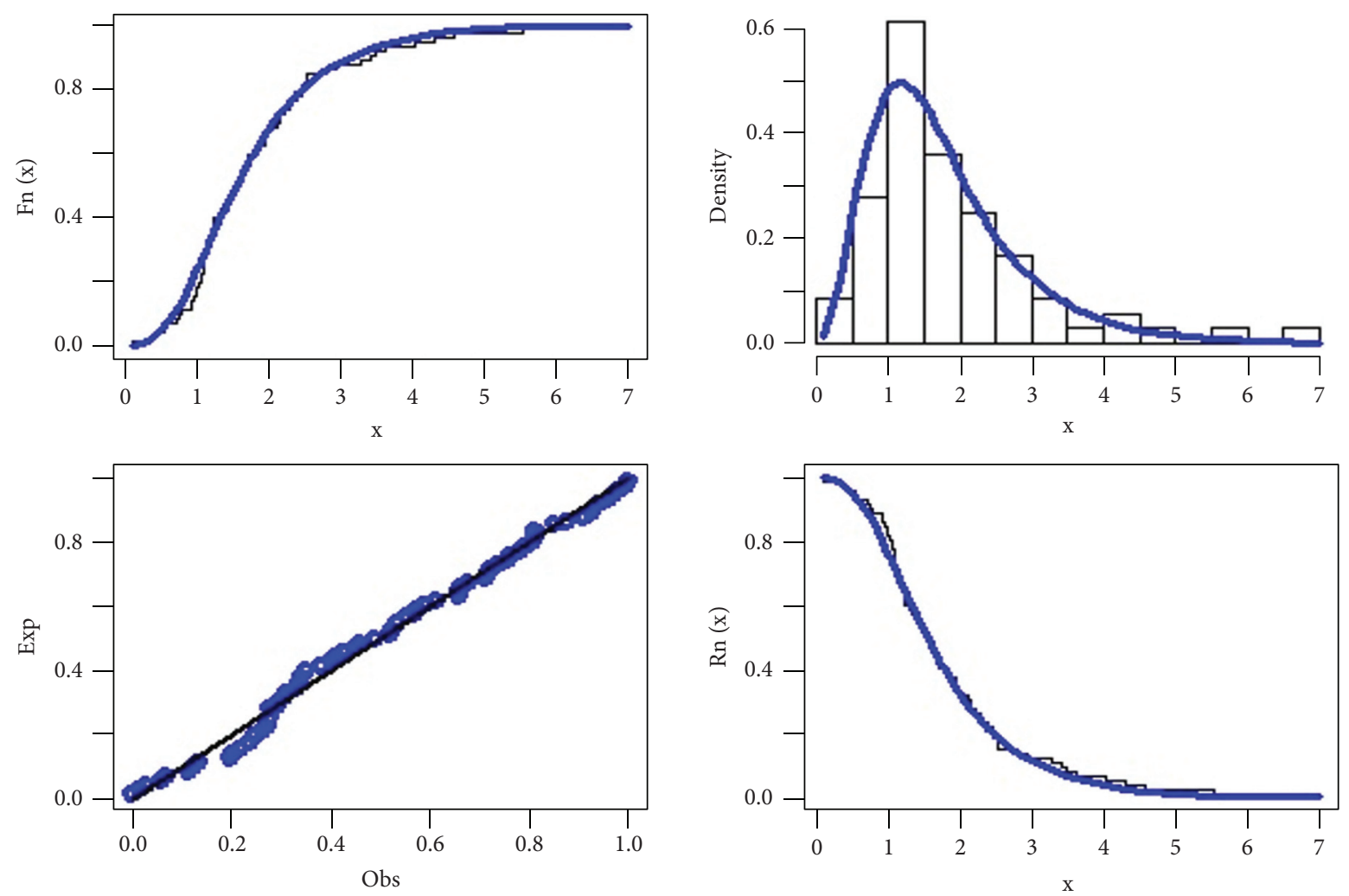

FIgURE 2: Fitted cdf, pdf, and pp plots for dataset I.
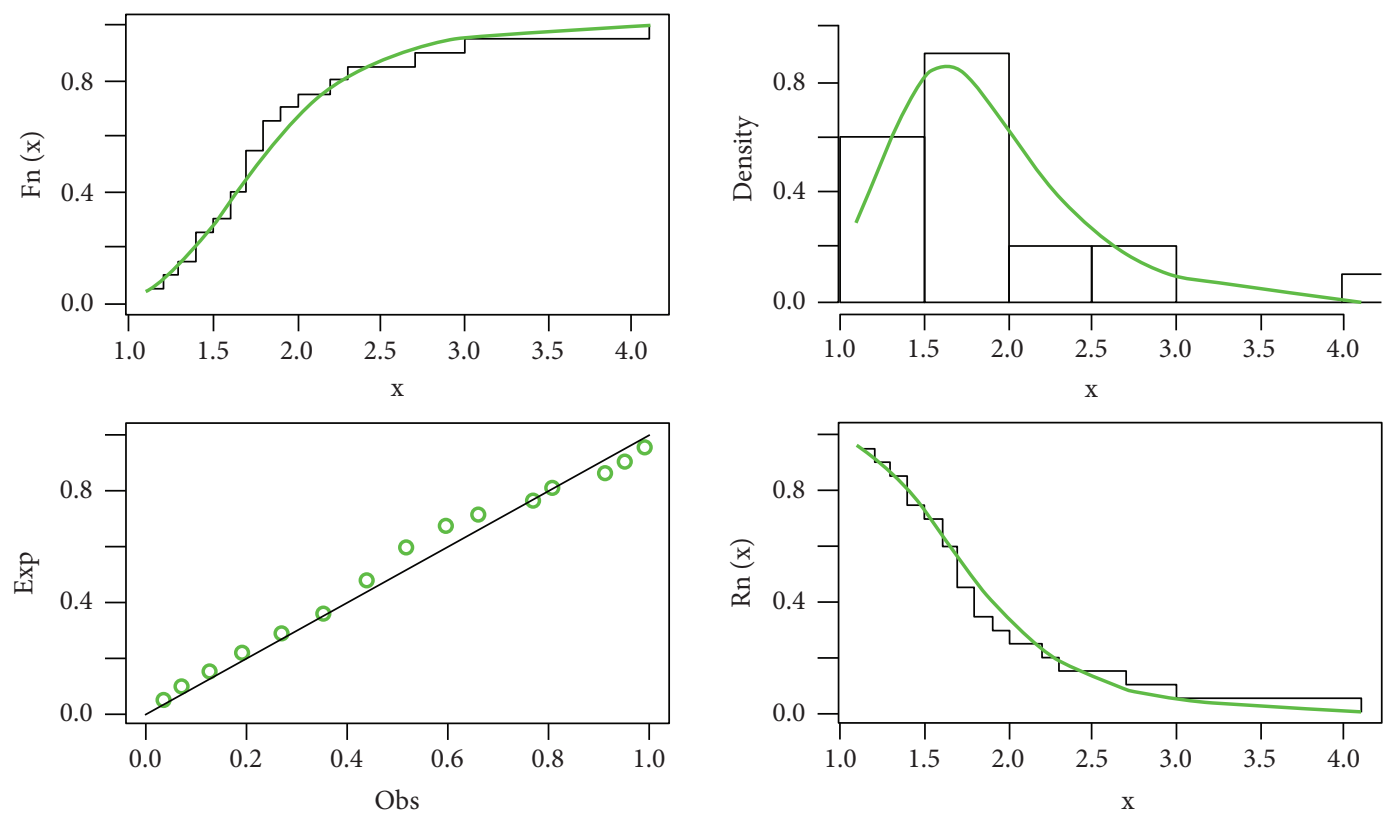

Figure 3: Fitted cdf, pdf, and pp plots for dataset II.

The goodness of fits of the TCPOFE model is compared with those of other competitive models, namely, Marshall-Olkin (MO) E (MOE), Burr X-E (BrXE), Kumaraswamy E $(\mathrm{KwE})$, generalized MO exponential (GMOE), beta exponential (BE), Kumaraswamy MO E (KMOE), MO Kumaraswamy $\mathrm{E}$ (MOKE), moment exponential (ME), and exponential (E) models.
The MLEs and standard errors (SEs) are calculated for both datasets. The numerical results are listed in Tables 2 and 3 . From the numerical values provided in Tables 4 and 5 and the information shown in Figures 2 and 3, the TCPOFE model performs considerably better than the other considered extensions of the E model. Hence, the TCPOFE model is a good alternative over these models for both datasets. 


\section{Conclusion}

In this article, we have shown how the use of the "truncated Cauchy power" scheme applied to the odd Fréchet family can lead to new flexible statistical models for the analysis of right-skewed data. Precisely, we have proceeded as follows. First, we have defined the TCPOF-G family of distributions, discussed the motivations behind it, and studied its main properties of interest. Four particular examples of the new family are proposed. Rényi entropy of the TCPOF is calculated. We used the maximum likelihood technique to estimate the parameters. Examples from real-world data demonstrate empirically the significance and promise of the proposed family. In the future, we are planning to use the new family to generate a new model and study its statistical properties and prove the flexibility of it by using more real datasets.

\section{Data Availability}

Please contact the relevant author if you would like to acquire the numerical dataset used to conduct the research described in the paper.

\section{Conflicts of Interest}

The authors declare that they have no conflicts of interest.

\section{Acknowledgments}

The authors extend their appreciation to the deputyship for research and innovation, Ministry of Education in Saudi Arabia, for funding this research work through project no. IFKSURG-1438-086.

\section{References}

[1] N. Eugene, C. Lee, and F. Famoye, "Beta-normal distribution and its applications," Communications in Statistics-Theory and Methods, vol. 31, no. 4, pp. 497-512, 2002.

[2] K. Zografos and N. Balakrishnan, "On families of beta and generalized gamma-generated distributions and associated inference," Statistical Methodology, vol. 6, no. 4, pp. 344-362, 2009.

[3] T. Zhang and M. Xie, "On the upper truncated weibull distribution and its reliability implications," Reliability Engineering \& System Safety, vol. 96, no. 1, pp. 194-200, 2011.

[4] H. Najarzadegan, M. H. Alamatsaz, and S. Hayati, "Truncated weibull-G more flexible and more reliable than beta-G distribution," International Journal of Statistics and Probability, vol. 6, no. 5, pp. 1-17, 2017.

[5] R. A. R. Bantan, F. Jamal, C. Chesneau, and M. Elgarhy, "Truncated inverted Kumaraswamy generated family of distributions with applications," Entropy, vol. 21, no. 11, p. 1089, 2019.

[6] M. A. Aldahlan, "Type II truncated Fréchet generated family of distributions," International Journal of Machine Intelligence and Applications, vol. 7, pp. 221-228, 2019.

[7] R. A. Bantan, F. Jamal, C. Chesneau, and M. Elgarhy, "Type II power topp-leone generated family of distributions with applications," Symmetry, vol. 12, no. 1, pp. 1-22, 2020.
[8] Z. Ahmad, M. Elgarhy, G. G. Hamedani, and N. S. Butt, "Odd generalized N-H generated family of distributions with application to exponential model," Pakistan Journal of Statistics and Operation Research, vol. 16, no. 1, pp. 53-71, 2020.

[9] S. Al-Marzouki, F. Jamal, C. Chesneau, and M. Elgarhy, "Topp-leone odd Fréhet generated family of distributions with applications to COVID-19 data sets," Computer Modeling in Engineering and Sciences, vol. 125, no. 1, pp. 437-458, 2020.

[10] M. M. Badr, I. Elbatal, F. Jamal, C. Chesneau, and M. Elgarhy, "The transmuted odd fréchet-G family of distributions: theory and applications," Mathematics, vol. 8, no. 6, pp. 958-978, 2020.

[11] R. A. R. Bantan, C. Chesneau, F. Jamal, I. Elbatal, and M. Elgarhy, "The truncated burr X-G family of distributions: properties and applications to actuarial and financial data," Entropy, vol. 23, no. 8, p. 1088, 2021.

[12] M. A. u. Haq and M. Elgarhy, "The odd fréchet-G family of probability distributions," Journal of Statistics Applications \& Probability, vol. 7, no. 1, pp. 189-203, 2018.

[13] P. Rider, "Generalized cauchy distributions," Annals of the Institute of Statistical Mathematics, vol. 9, no. 1, pp. 215-223, 1957.

[14] S. Nadarajah and S. Kotz, "A truncated Cauchy distribution," International Journal of Mathematical Education in Science \& Technology, vol. 37, no. 5, pp. 605-608, 2006.

[15] J. Ohakwe and B. Osu, "The existence of the moments of the Cauchy distribution under a simple transformation of dividing with a constant," Journal of Theoretical Mathematics and Applications, vol. 1, pp. 27-35, 2011.

[16] E. Jacob and K. Jayakumar, "On half-cauchy distribution and process," International Journal of Statistika and Mathematika, vol. 3, pp. 77-81, 2012.

[17] G. Hamedani and I. Ghosh, "Kumaraswamy-half-cauchy distribution: characterizations and related results," International Journal of Statistics and Probability, vol. 4, pp. 94-100, 2015.

[18] E. Alshawarbeh, F. Famoye, and C. Lee, "Beta-cauchy distribution: some properties and applications," Journal of Statistical Theory and Applications, vol. 12, no. 4, pp. 378-391, 2013.

[19] M. Zubair, M. H. Tahir, G. M. Cordeiro, A. Alzaatreh, E. M. M. Ortega, and M. Ortega, "The power-cauchy negativebinomial: properties and regression," Journal of Statistical Distributions and Applications, vol. 5, no. 1, pp. 1-17, 2018.

[20] M. Aldahlan, F. Jamal, C. Chesneau, M. Elgarhy, and I. Elbatal, "The truncated cauchy power family of distributions with inference and applications," Entropy, vol. 22, no. 346, pp. 1-24, 2019.

[21] T. Bjerkedal, "Acquisition of resistance in guinea pigs infected with different doses of virulent tubercle Bacilli1," American Journal of Epidemiology, vol. 72, no. 1, pp. 130-148, 1960.

[22] A. J. Gross and V. A. Clark, Survival Distributions: Reliability Applications in the Biomedical Sciences, John Wiley \& Sons, New York, NY, USA, 1975. 